Annales Academiæ Scientiarum Fennicæ

Mathematica

Volumen 35, 2010, 335-350

\title{
HOPF DECOMPOSITION AND HOROSPHERIC LIMIT SETS
}

\author{
Vadim A. Kaimanovich \\ University of Ottawa, Department of Mathematics and Statistics \\ 585 King Edward Ave, Ottawa ON, K1N 6N5, Canada; vkaimano@uottawa.ca
}

\begin{abstract}
By looking at the relationship between the recurrence properties of a countable group action with a quasi-invariant measure and the structure of the space of ergodic components, we establish a simple general description of the Hopf decomposition of the action into its conservative and dissipative parts in terms of the Radon-Nikodym derivatives. As an application we describe the conservative part of the boundary action of a discrete group of isometries of a Gromov hyperbolic space with respect to an invariant quasi-conformal stream.
\end{abstract}

Conservativity and dissipativity are, along with ergodicity, the most basic notions of ergodic theory which go back to its mechanical and thermodynamical origins. The famous Poincaré recurrence theorem states that any measure preserving transformation $T$ of a probability space $(X, m)$ is conservative in the sense that any positive measure subset $A \subset X$ is recurrent, i.e., for a.e. starting point $x \in A$ the trajectory $\left\{T^{n} x\right\}$ eventually returns to $A$. These definitions clearly make sense for any measure class preserving action $G \circlearrowleft(X, m)$ of a general countable group $G$ on a probability space. The opposite notions are those of dissipativity and of a wandering set, i.e., such a set $A$ that all its translates $g A, g \in G$, are pairwise disjoint. An action is called dissipative if it admits a wandering set of positive measure, and it is called completely dissipative if, moreover, there is a wandering set such that the union of its translates is $(\bmod 0)$ the whole action space.

Our approach to these properties is based on the observation that the notions of conservativity and dissipativity admit a very natural interpretation in terms of the ergodic decomposition of the action (under the assumption that such a decomposition exists, i.e., that the action space is a Lebesgue measure space). Let $\mathscr{C} \subset X$ denote the union of all the purely non-atomic ergodic components, and let $\mathscr{D}=X \backslash \mathscr{C}$ be the union of all the purely atomic ergodic components. We call $\mathscr{C}$ and $\mathscr{D}$ the continual and discontinual parts of the action, respectively. Further, let $\mathscr{D}_{\mathbf{1}}$ (resp., $\mathscr{D}_{>\mathbf{1}}$ ) be the subset of $\mathscr{D}$ consisting of the points with trivial (resp., non-trivial) stabilizers, i.e., the union of free (resp., non-free) orbits in $\mathscr{D}$. The restriction of the action to the set Cons $=\mathscr{C} \cup \mathscr{D}_{>1}$ is conservative, whereas the restriction to the set Diss $=\mathscr{D}_{1}$ is completely dissipative, thus providing the so-called Hopf decomposition of the action space into the conservative and completely dissipative parts (Theorem 14).

doi:10.5186/aasfm.2010.3522

2000 Mathematics Subject Classification: Primary 37A20; Secondary 22F10, 28D99, 30F40, $53 \mathrm{C} 20$.

Key words: Conservative action, dissipativity, recurrent set, wandering set, Hopf decomposition, ergodic components, Gromov hyperbolic space, horospheric limit set.

This paper was mostly written during my stay at the Erwin Schrödinger Institute (ESI) in Vienna as a senior research fellow. I would like to thank ESI for the support and excellent working conditions. The research was undertaken, in part, thanks to funding from the Canada Research Chairs program. 
[Historically, such a decomposition was first established in the pioneering paper of Eberhard Hopf [Hop30] for one-parameter groups of measure preserving transformations.] Moreover, the restriction of the action to the set Cons $_{\infty}=\mathscr{C} \cup \mathscr{D}_{\infty}$ (where $\mathscr{D}_{\infty}$ is the set of points from $\mathscr{D}$ with infinite stabilizers) is infinitely conservative in the sense that a.e. orbit passing thorugh this set returns to it infinitely often. These descriptions are also valid if one defines conservativity and infinite conservativity in terms of translates of positive measure sets. Namely, for any positive measure subset of Cons (resp., of Cons $)_{\infty}$ ) there exists $g \in G \backslash\{e\}$ (resp., infinitely many such $g$ ) with $m(A \cap g A)>0$.

Although these facts are definitely known to the specialists (the important particular cases of $\mathbf{Z}$-actions and of free actions are considered in [Kre85] and [Aar97], respectively), they rather belong to the "folklore" (e.g., see the discussion in [Tuk97]), and the treatment of this issue in the literature is sometimes confused, so that we felt it necessary to give a clear and concise proof.

The continual part $\mathscr{C}$ can be described as the set of points for which the orbitwise sum of the Radon-Nikodym derivatives of the action is infinite (Theorem 23(iii) and Corollary 24). Actually, this description is also valid in the full generality of discrete equivalence relations (Remark 28). Once again, the specialists in the latter theory will hardly be surprised by this result. However, to the best of our knowledge, in spite of its simplicity it has never been formulated explicitly.

In the case of $(\bmod 0)$ free actions Cons $=$ Cons $_{\infty}=\mathscr{C}$, so that in this situation the above condition completely characterizes the conservative and the infinitely conservative parts of the action. A simple consequence of this fact is the description of the infinitely conservative part Cons $_{\infty}$ of an arbitrary action $G \circlearrowleft(X, m)$ as the set of points $x \in X$ with the property that

$$
\text { there exists } t=t(x) \text { such that }\{g \in G: d g m / d m(x) \geq t\} \text { is infinite }
$$

(Theorem 29).

The latter result completely trivializes the problem of a geometric description of the Hopf decomposition of the boundary action of a discrete group of isometries $G$ of a Gromov hyperbolic space $\mathscr{X}$ with respect to a certain natural measure class, which is our main application.

More precisely, for any boundary point $\omega \in \partial \mathscr{X}$ and any $x, y \in X$ let $\beta_{\omega}(x, y)=$ $\lim \sup _{z}[d(y, z)-d(x, z)]$, where $z \in \mathscr{X}$ converges to $\omega$ in the hyperbolic compactification. For CAT $(-1)$ spaces $\beta_{\omega}$ are the usual Busemann cocycles, whereas in the general case the cocycle identity is satisfied up to a uniformly bounded additive error only, so that we have to call them Busemann quasi-cocycles. Then one can look for a family $\lambda=\left\{\lambda_{x}\right\}$ of finite pairwise equivalent boundary measures parameterized by points $x \in \mathscr{X}$ (following [KL05] we use the term stream for such families) whose mutual Radon-Nikodym derivatives are prescribed by $\beta_{\omega}$ in the sense that

$$
\left|\log \frac{d \lambda_{x}}{d \lambda_{y}}(\omega)-D \beta_{\omega}(x, y)\right| \leq C \quad \forall x, y \in \mathscr{X}, \omega \in \partial \mathscr{X}
$$

for certain constants $C \geq 0, D>0$. We call such a stream quasi-conformal of dimension $D$. It is invariant if $g \lambda_{x}=\lambda_{g x}$ for any $g \in G, x \in \mathscr{X}$.

In the CAT $(-1)$ case any invariant quasi-conformal stream is equivalent (with uniformly bounded Radon-Nikodym derivatives) to an invariant conformal stream of the same dimension, i.e., such that the logarithms of the Radon-Nikodym derivatives 
are precisely proportional to the Busemann cocycles. Given a reference point $o \in$ $\mathscr{X}$, an invariant (quasi-)conformal stream is uniquely determined just by a (quasi-) conformal measure $\lambda_{o}$ with the property that $\log d g \lambda_{o} / d \lambda_{o}(\omega)$ is proportional to $\beta_{\omega}(g o, o)$ (up to a uniformly bounded additive error).

Coornaert [Coo93] proved (applying the construction used by Patterson [Pat76] in the case of Fuchsian groups) that for any discrete group of isometries $G$ of a Gromov hyperbolic space $\mathscr{X}$ with a finite critical exponent $D$ there exists an invariant quasiconformal stream of dimension $D$ supported by the limit set of $G$.

It is with respect to the measure class of an invariant quasi-conformal stream $\lambda=$ $\left\{\lambda_{x}\right\}$ that we study the Hopf decomposition of the boundary action. The geometric description $(* *)$ of the Radon-Nikodym derivatives in combination with criterion (*) immediately implies that the infinitely conservative part Cons $s_{\infty}$ of the boundary action coincides $(\bmod 0)$ with the big horospheric limit set $\Lambda_{G}^{\text {horB }}$ of the group $G$, i.e., with the set of points $\omega \in \partial \mathscr{X}$ for which there exists $t=t(\omega)$ such that $\{g \in G$ : $\left.\beta_{\omega}(g o, o) \geq t\right\}$ is infinite for a certain fixed reference point $o \in X$, or, in other words, with the set of points $\omega \in \partial \mathscr{X}$ such that a certain horoball centered at $\omega$ contains infinitely many points from the orbit $G o$ (Theorem 40). The conservative part Cons of the action is then the union of $\Lambda_{G}^{\text {horB }} \cong$ Cons $_{\infty}$ and the set of $\partial \mathscr{X}_{>1}$ of all boundary points with non-trivial $G$-stabilizers (note that the set $\partial \mathscr{X}_{\infty}$ of boundary points with infinite stabilizers is automatically contained in $\Lambda_{G}^{\text {horB }}$ ).

This characterization of the conservative part of the boundary action was first established by Pommerenke [Pom76] for Fuchsian groups with respect to the visual stream on the boundary circle (although in a somewhat different terminology). Pommerenke's argument uses analytic properties of the Blaschke products and does not immediately carry over to the higher dimensional situation. Sullivan [Sul81] used a more direct geometrical approach and proved this characterization for Kleinian groups, again with respect to the visual stream. Actually he considered the small horospheric limit set $\Lambda_{G}^{\text {horS }}$ (also called just horospheric limit set; it is defined by requiring that the intersection of any horoball centered at $\omega \in \Lambda_{G}^{\text {hors }}$ with the orbit $G o$ be infinite) essentially showing that $\Lambda^{\text {horB }} \backslash \Lambda^{\text {horS }}$ is a null set. By extending Sullivan's approach (with some technical complications) Tukia [Tuk97] proved Theorem 40 for Kleinian groups with respect to an arbitrary invariant conformal stream. Our completely elementary approach subsumes all these particular cases; note that in all of them the set of boundary points with non-trivial stabilizers is negligible, so that Cons $=$ Cons $_{\infty}$ (which is not necessarily the case in general).

By the way, concerning stabilizers of boundary points it would be interesting to know whether the set of boundary points with infinite $G$-stabilizers $\partial \mathscr{X}_{\infty}$ (or, equivalently, the set of boundary points whose stabilizer is an infinite torsion group) may be at all uncountable (let alone be non-negligible with respect to an invariant quasi-conformal stream). This option seems to be quite unlikely, see Remark 43.

It is clear from looking at criterion $(*)$ that the right object in the context of studying conservativity of boundary actions is the big horospheric limit set $\Lambda^{\text {horB }}$ rather than the small one $\Lambda^{\text {hors }}$. Nonetheless it is plausible that $\Lambda^{\text {horB }} \backslash \Lambda^{\text {horS }}$ is a null set with respect to any invariant quasi-conformal stream on an arbitrary Gromov hyperbolic space. This was proved by Sullivan [Sul81] for Kleinian groups with respect to the visual stream, and for subgroups of a free group (again with respect to the uniform stream) it was done in [GKN09], see Remark 45. 
We refer the reader to [GKN09] for a recent detailed study of the interrelations between various kinds of limit sets in the simplest model case of the action of a free subgroup on the boundary of an ambient finitely generated free group. Actually, it was this collaboration that brought me to the issues discussed in the present article, and I would like to thank my collaborators Rostislav Grigorchuk and Tatiana Nagnibeda for the gentle insistence with which they encouraged my work. I am also grateful to an anonymous referee for pointing out an oversight related to elliptic elements in the first version of the paper. The ensuing revision led to a much better understanding of the Hopf decomposition for non-free actions.

\section{Structure of the ergodic components and recurrence properties}

1.A. Lebesgue spaces. We begin by recalling the basic properties of the Lebesgue measure spaces introduced by Rokhlin, see [Roh52, CFS82]. Measuretheoretically these are the probability spaces such that their non-atomic part is isomorphic to an interval with the Lebesgue measure on it. Thus, any Lebesgue space is uniquely characterized by its signature $\sigma=\left(\sigma_{0} ; \sigma_{1}, \sigma_{2}, \ldots\right)$, where $\sigma_{0}$ is the total mass of the non-atomic part, and $\sigma_{1} \geq \sigma_{2} \geq \ldots$ is the ordered sequence of the values of its atoms (extended by zeroes if the set of atoms is finite or empty). There is also an intrinsic definition of the Lebesgue spaces based on their separability properties. However, for applications it is usually enough to know that any Polish topological space (i.e., separable and completely metrizable) endowed with a Borel probability measure is a Lebesgue measure space. We shall follow the standard measure theoretical convention:

Unless otherwise specified, all the identities, properties etc. related to measure spaces will be understood mod 0 (i.e., up to null sets). In particular, all the $\sigma$-algebras are assumed to be complete, i.e., to contain all the measure 0 sets.

An important feature of the Lebesgue measure spaces is

Theorem E. (Existence of conditional probabilities) Let $p:(X, m) \rightarrow(\bar{X}, \bar{m})$ be a homomorphism (projection, factorization, quotient map) of Lebesgue spaces, i.e., for any measurable set $\bar{A} \subset \bar{X}$ its preimage $A=p^{-1}(\bar{A}) \subset X$ is also measurable, and $m(A)=\bar{m}(\bar{A})$. Then the preimages $X_{\bar{x}}=p^{-1}(\bar{x}), \bar{x} \in \bar{X}$, can be uniquely endowed with conditional probability measures $m_{\bar{x}}$ in such a way that $\left(X_{\bar{x}}, m_{\bar{x}}\right)$ are Lebesgue spaces and the measure $m$ decomposes into an integral of the measures $m_{\bar{x}}, \bar{x} \in$ $\bar{X}$, with respect to the quotient measure $\bar{m}$ on $\bar{X}$. Namely, for any function $f \in$ $L^{1}(X, m)$ its restrictions $f_{\bar{x}}$ to $X_{\bar{x}}$ are measurable and belong to the respective spaces $L^{1}\left(X_{\bar{x}}, m_{\bar{x}}\right)$, the integrals $\bar{f}(\bar{x})=\left\langle f_{\bar{x}}, m_{\bar{x}}\right\rangle$ depend on $\bar{x}$ measurably, and $\langle f, m\rangle=$ $\langle\bar{f}, \bar{m}\rangle$ (cf. the classical Fubini theorem).

In fact, the above property follows from the classical Fubini theorem in view of Rokhlin's

Theorem C. (Classification of homomorphisms of Lebesgue spaces) Any homomorphism $p:(X, m) \rightarrow(\bar{X}, \bar{m})$ of Lebesgue spaces is uniquely (up to an isomorphism) determined by the signatures of the quotient measure $\bar{m}$ and of the conditional measures $m_{\bar{x}}$. Namely, let us denote by $I$ the unit interval endowed with the Lebesgue measure $\lambda$, and partition $I$ into a union of consecutive intervals $I_{0}, I_{1}, \ldots$ with $\lambda\left(I_{i}\right)=\sigma_{i}$ for a certain signature $\sigma$. Further, let us consider a coordinate-wise 
measurable assignment of signatures $\sigma^{x}$ to points $x \in I$ which is constant on the intervals $I_{1}, I_{2}, \ldots$, and, as before, let $I_{0}^{x}, I_{1}^{x}, \ldots$ be the consecutive subintervals of $I$ with $\lambda\left(I_{i}^{x}\right)=\sigma_{i}^{x}$. Denote by $(X, m)$ the Lebesgue space obtained from the square $(I \times I, \lambda \otimes \lambda)$ by collapsing the sets $\{x\} \times I_{i}^{x}, i \geq 1, x \in I_{0}$, and $I_{j} \times I_{i}^{x}, i, j \geq 1, x \in I_{j}$, onto single points. In the same way, let $(\bar{X}, \bar{m})$ be the quotient space of the interval $(I, \lambda)$ obtained by collapsing the intervals $I_{1}, I_{2}, \ldots$ onto single points, so that the signature of $(\bar{X}, \bar{m})$ is $\sigma$. Then the projection of the square $I \times I$ onto the first coordinate gives rise to a homomorphism from $(X, m)$ to $(\bar{X}, \bar{m})$, and the signatures of the associated conditional measures are precisely $\sigma^{x}$. The claim is that any homomorphism of Lebesgue spaces can be obtained in this way. In particular, if both the quotient measure $\bar{m}$ and all the conditional measures $m_{\bar{x}}$ are purely non-atomic (i.e., have the signature $(1 ; 0,0, \ldots))$, then the corresponding quotient map is isomorphic just to the projection of the unit square onto the first coordinate.

Obviously, any homomorphism of Lebesgue spaces gives rise to the preimage $s u b-\sigma$-algebra in $X$ which consists of the preimages of all the measurable sets in $\bar{X}$. Another important feature of the Lebesgue spaces is that, in fact, an arbitrary sub$\sigma$-algebra in $X$ can be obtained in this way for a certain uniquely defined quotient map.

Below we shall use the following elementary fact which follows at once from the uniqueness of the system of conditional measures.

Lemma 1. Let $T$ be an invertible measure class preserving transformation of a Lebesgue space $(X, m)$, and let $p:(X, m) \rightarrow(\bar{X}, \bar{m})$ be its $T$-invariant projection, i.e., $p(T x)=p(x)$ for a.e. $x \in X$. Then the conditional measures $m_{\bar{x}}, \bar{x} \in \bar{X}$, of the projection $p$ are quasi-invariant with respect to $T$ and have the same Radon-Nikodym derivatives as the measure $m$ :

$$
\frac{d T m}{d m}(x)=\frac{d T m_{\bar{x}}}{d m_{\bar{x}}}(x), \quad \text { where } \bar{x}=p(x),
$$

for a.e. $x \in X$.

1.B. Ergodic components, continuality and discontinuality. Let now $G \circlearrowleft(X, m)$ be an action of an infinite countable group $G$ by measure class preserving transformations on a Lebesgue space $(X, m)$ — which will be our standing assumption through the rest of this Section.

The quotient space $(\bar{X}, \bar{m})$ of $(X, m)$ determined by the $\sigma$-algebra of $G$-invariant sets is called the space of ergodic components of the action of $G$ on the space $(X, m)$, and the preimages $X_{\bar{x}}$ endowed with the conditional measures $m_{\bar{x}}$ are called ergodic components. The ergodic components $X_{\bar{x}}$ are $G$-invariant, the conditional measures $m_{\bar{x}}$ are $G$-quasi-invariant, and the action of $G$ on the spaces $\left(X_{\bar{x}}, m_{\bar{x}}\right)$ is ergodic (e.g., see [Sch77]). Lemma 1 implies that the conditional measures $m_{\bar{x}}$ on the ergodic components have the same Radon-Nikodym derivatives with respect to the action of $G$ as the original measure $m$.

Since the ergodic components are ergodic, each of them is either purely atomic (in which case it consists of a single $G$-orbit), or purely non-atomic.

Definition 2. The continual $\mathscr{C}$ (resp., discontinual $\mathscr{D}$ ) part of the action $G \circlearrowleft$ $(X, m)$ is the union of all the purely non-atomic (resp., purely atomic) components of the action. Denote by $\overline{\mathscr{C}}$ and $\overline{\mathscr{D}}$ the corresponding subsets of the space of ergodic 
components $\bar{X}$ (their measurability follows from Theorem C). An orbit Gx is continual (resp., discontinual) if it belongs to $\mathscr{C}$ (resp., to $\mathscr{D}$ ). The action is discontinual if $m(\mathscr{D})>0$ and continual otherwise. If $m(\mathscr{C})=0$ the action is called completely discontinual.

Remark 3. The quotient measure class on the space of ergodic components and the measure classes of the conditional measures on the ergodic components do not change when the measure $m$ is replaced with an equivalent one, so that Definition 2 (as well as various definitions below related to conservativity and dissipativity) depends only on the measure class of $m$.

Lemma 4. Let $A \subset X$ be a measurable $G$-invariant subset. It is contained in the discontinual part $\mathscr{D}$ if and only if one can select, in a measurable way, a representative from each $G$-orbit contained in $A$, i.e., if and only if there exists a measurable map $\pi: A \rightarrow A$ which is constant along the orbits of the action.

Proof. If $\pi$ is such a map, then it identifies the space of ergodic components of $A$ with $\pi(A)$, so that in particular $A \subset \mathscr{D}$. Conversely, Theorem $\mathrm{C}$ readily provides a map $\pi: \mathscr{D} \rightarrow \mathscr{D}$ with the required properties.

Remark 5. Below we shall also encounter the situation when instead of a map with the properties from the above Lemma one has an orbit constant measurable map $x \mapsto M_{x}$, where $M_{x}$ is a non-empty finite subset of the orbit $G x$. This situation can be easily reduced to Lemma 4 by choosing (in a measurable way!) just a single point from each of the sets $M_{x}$. This can be done, for instance, by identifying the space $(X, m)$ with the unit interval (with possible collapsing corresponding to the atoms of the measure $m$ ) and taking then the minimal of the points of $M_{x}$.

1.C. Wandering and recurrent sets. The definitions below are well-known (e.g., see [Kre85, Aar97]). However, we had to slightly modify them (and, in particular, to distinguish recurrence from infinite recurrence) in order to take into account certain effects which do not arise for the usually considered free and $\mathbf{Z}$-actions.

We shall begin with the following measure-theoretical analogue of the topological notion of a fundamental domain.

Definition 6. A measurable subset $A \subset X$ is called wandering if all its translates $\{g A\}_{g \in G}$ are pairwise disjoint.

Given a measurable $G$-invariant subset $A \subset X$, denote by $A_{\mathbf{k}}, k=1,2, \ldots, \infty$, the set of all points $x \in X$ whose stabilizer $G_{x}$ has size $k$ (obviously, all the sets $A_{\mathbf{k}}$ are $G$-invariant and measurable along with the set $A$ ). Also denote by $A_{>\mathbf{1}}=\bigcup_{k>1} A_{\mathbf{k}}$ the set of all points from $A$ with non-trivial (possibly infinite) stabilizers and by $A_{<\infty}=\bigcup_{k<\infty} A_{\mathbf{k}}$ the set of all points from $A$ with finite (including trivial) stabilizers.

Proposition 7. Any wandering set is contained in $\mathscr{D}_{\mathbf{1}}$, and there is a maximal wandering set, i.e., a wandering set $A$ such that $\mathscr{D}_{1}=\bigcup_{g \in G} g A$.

Proof. If $A$ is a wandering set, then the map $\pi$ from the $G$-invariant union $\widetilde{A}=\bigcup_{g \in G} g A$ to $A$ defined as $\pi(x)=G x \cap A$ is measurable and $G$-invariant, so that $A \subset \mathscr{D}$ by Lemma 4. Moreover, all the orbits intersecting $A$ are obviously free, whence $A \subset \mathscr{D}_{\mathbf{1}}$.

As for the maximality, one can take for such a wandering set any measurable section of the projection $\mathscr{D}_{\mathbf{1}} \rightarrow \overline{\mathscr{D}}_{\mathbf{1}}$ (here $\overline{\mathscr{D}}_{\mathbf{1}}$ is the subset of the space of ergodic 
components $\bar{X}$ corresponding to the $G$-invariant measurable set $\mathscr{D}_{\mathbf{1}}$; the existence of such a section follows from Lemma 4).

Remark 8. A proof of existence of a maximal wandering set valid without assuming that $(X, m)$ is a Lebesgue space is given in [Aar97, Proposition 1.6.1].

The notion of a recurrent set is opposite to that of a wandering set.

Definition 9. A measurable set $A \subset X$ is called recurrent (resp., infinitely recurrent) if for a.e. point $x \in A$ the trajectory $G x$ eventually returns to $A$, i.e., $g x \in A$ for a certain element $g \in G$ other than the group identity $e$ (resp., returns to $A$ infinitely often, i.e., $g x \in A$ for infinitely many elements $g \in G$ ).

Proposition 10. Any non-trivial measurable subset of the continual part $\mathscr{C}$ is infinitely recurrent.

Proof. For a measurable subset $A \subset \mathscr{C}$ put

$$
A_{0}=\{x \in A: g x \in A \text { for finitely many } g \in G\} .
$$

Then obviously $A_{0} \subset F$, where

$$
F=\{x \in X: G x \cap A \text { is non-empty and finite }\} .
$$

The set $F$ is $G$-invariant and measurable, and by Lemma 4 and Remark $5 F \subset \mathscr{D}$. Therefore $A_{0}$ must be a null set, whence the claim.

The definition of the sets $\mathscr{D}_{>1}$ and $\mathscr{D}_{\infty}$ also immediately implies

Proposition 11. Any non-trivial measurable subset of $\mathscr{D}_{>1}$ (resp., of $\mathscr{D}_{\infty}$ ) is recurrent (resp., infinitely recurrent).

There is another definition of recurrence based on considering translates of positive measure subsets rather than orbits of individual points (it is used, for instance, in [Sul81, Tuk97]). However, the recurrence properties of subsets of $X$ established in Proposition 10 and Proposition 11 hold for this definition as well:

Proposition 12. For any non-trivial measurable subset $A$ of $\mathscr{C} \cup \mathscr{D}_{>\mathbf{1}}$ (resp., of $\mathscr{C} \cup \mathscr{D}_{\infty}$ ) there exists $g \in G \backslash\{e\}$ with $m(A \cap g A)>0$ (resp., there exist infinitely many such $g$ ).

Proof. (i). If all the translates of a set $A \subset \mathscr{C} \cup \mathscr{D}_{>1}$ are pairwise disjoint, then $A$ is a wandering set, and therefore $A \subset \mathscr{D}_{\mathbf{1}}$ by Proposition 7 , whence a contradiction.

(ii). Given a set $A \subset \mathscr{C} \cup \mathscr{D}_{\infty}$, let $\mathscr{R}_{x}=\{g \in G: g x \in A\}$ for $x \in A$. Obviously, $m(A \cap g A)>0$ if an only if $m\left\{x \in A: g \in \mathscr{R}_{x}\right\}>0$. Thus, if $m(A \cap g A)>0$ only for finitely many $g \in G$, then the sets $\mathscr{R}_{x}$ must be finite for a.e. $x \in A$, which is impossible in view of Proposition 10 and Proposition 11.

\section{D. Hopf decomposition.}

Definition 13. An action $G \circlearrowleft(X, m)$ is called conservative (resp., infinitely conservative) if any measurable subset $A \subset X$ is recurrent (resp., infinitely recurrent). It is called dissipative if there is a non-trivial wandering set, and completely dissipative if the whole action space $X$ is the union of translates of a certain wandering set.

Taking stock of the Propositions from Section 1.C we obtain 
Theorem 14. (Hopf decomposition for general actions) Let $G \circlearrowleft(X, m)$ be an action of an infinite countable group $G$ by measure class preserving transformations on a Lebesgue space $(X, m)$. Then

(i) The action space decomposes into the disjoint union of $G$-invariant measurable sets Cons $=\mathscr{C} \cup \mathscr{D}_{>1}$ and Diss $=\mathscr{D}_{1}$ (called its conservative and dissipative parts, respectively) such that the restriction of the action to Cons is conservative and the restriction to Diss is completely dissipative.

(ii) The restriction of the action to the $G$-invariant measurable set Cons $_{\infty}=$ $\mathscr{C} \cup \mathscr{D}_{\infty}$ (called the infinitely conservative part of the action) is infinitely conservative.

(iii) The restriction of the action to the set Cons $\boldsymbol{\infty}_{\mathbf{0}} \backslash$ Cons $=\bigcup_{1<k<\infty} \mathscr{D}_{\mathbf{k}}$ is conservative, but neither infinitely conservative nor dissipative.

Corollary 15. Cons $=$ Cons $_{\infty} \cup X_{>1}$.

Corollary 16. If the action $G \circlearrowleft(X, m)$ is free, i.e., $\mathscr{D}=\mathscr{D}_{\mathbf{1}}$, then Cons $=$ Cons $_{\infty}=\mathscr{C}$, and Diss $=\mathscr{D}$.

Corollary 17. (Poincaré recurrence theorem) If the measure $m$ is invariant, then $\mathscr{D}_{1}$ is a null set, and therefore the action is conservative.

Remark 18. The decomposition described in Theorem 14(i) is unique. Indeed, let $X=C \sqcup D=C^{\prime} \sqcup D^{\prime}$ be two such decompositions. If they are different, then one of the sets $C \cap D^{\prime}, C^{\prime} \cap D$ must be non-empty. Let it be, for instance, $A=C \cap D^{\prime}$. Then the restriction of the action to the $G$-invariant set $A$ has to be simultaneously conservative (because $A \subset C$ ) and completely dissipative (because $A \subset D^{\prime}$ ), which is impossible.

Remark 19. Proposition 12 implies that Theorem 14 is also valid for the definitions of conservativity and infinite conservativity based on considering translates of positive measure sets.

Remark 20. If one defines strict recurrence and strict conservativity by requiring that the orbit $G x$ returns to the set $A$ at an orbit point different from the starting point $x$, then the strictly conservative and the strictly infinitely conservative parts of the action both coincide just with the continual part $\mathscr{C}$ (see Remark 28 below).

Remark 21. The group $\mathbf{Z}$ does not contain non-trivial finite subgroups, so that for its actions $\mathscr{D}_{<\infty}=\mathscr{D}_{1}$, and therefore in this case Cons $=$ Cons $_{\infty}=\mathscr{C} \cup \mathscr{D}_{\infty}$.

Remark 22. When dealing with the actions of the group $\mathbf{Z}$ one sometimes defines the notion of recurrence by looking only at the "positive semi-orbits" $\mathbf{Z}_{+} x$. All measurable subsets of $\mathscr{C}$ are infinitely recurrent in this sense as well. Indeed, for a subset $A \subset \mathscr{C}$ let $A_{0}=\left\{x \in A: \mathbf{Z}_{+} x \cap A\right.$ is finite $\}$. Then $x \mapsto z x$, where $z$ is the maximal element of $\mathbf{Z}$ with $z x \in A$, is a measurable "selection map" in the sense of Lemma 4 , so that $A_{0} \subset \mathscr{D}$, whence $A_{0}$ is a null set.

1.E. Continuality, conservativity and Radon-Nikodym derivatives. We shall now discuss a link between continuality, conservativity and the Radon-Nikodym derivatives of the action. Let us first consider the case of free actions.

Theorem 23. Let $G \circlearrowleft(X, m)$ be a free measure class preserving action of a countable group $G$ on a Lebesgue space. Denote by $\mu_{x}, x \in X$, the measure on the 
orbit $G x$ defined as

$$
\mu_{x}(g x)=\frac{d g^{-1} m}{d m}(x)=\frac{d m(g x)}{d m(x)}
$$

(obviously, the measures $\mu_{x}$ corresponding to different points $x$ from the same $G$-orbit are proportional). Then for a.e. point $x \in X$ the following conditions are equivalent:

(i) The orbit $G x$ is dissipative;

(ii) The orbit $G x$ is discontinual;

(iii) The measure $\mu_{x}$ is finite;

(iv) For any $t>0$ the set $\left\{y \in G x: \mu_{x}(y) \geq t\right\}$ is finite;

(v) The set $M_{x}$ of maximal weight atoms of the measure $\mu_{x}$ is non-empty and finite.

Proof. (i) $\Longleftrightarrow$ (ii). This is Corollary 16 .

(ii) $\Longrightarrow$ (iii). By Definition 2, the orbit $G x$ is discontinual if and only if it is an ergodic component of the $G$-action on $X$. By Lemma 1 in this case the measure $\mu_{x}$ is proportional to the conditional measure on this ergodic component, and therefore it is finite.

(iii) $\Longrightarrow$ (iv) $\Longrightarrow(\mathrm{v})$. Obvious.

(v) $\Longrightarrow$ (ii). Follows from Lemma 4 and Remark 5 (because the map $x \mapsto M_{x}$ is measurable in view of Theorem $\mathrm{C}$ ).

Corollary 24. Under conditions of Theorem 23

$$
\mathscr{C}=\text { Cons }=\left\{x \in X: \sum_{g \in G} \frac{\operatorname{dgm}(x)}{d m(x)}=\infty\right\}
$$

and

$$
\mathscr{D}=\text { Diss }=\left\{x \in X: \sum_{g \in G} \frac{d g m(x)}{d m(x)}<\infty\right\} .
$$

Remark 27. Condition (iv) from Theorem 23 is not, generally speaking, equivalent just to existence of $t>0$ such that the set $\left\{y \in G x: \mu_{x}(y) \geq t\right\}$ is finite (i.e., to boundedness of the values of the weights of the measure $\mu_{x}$ ). The most manifest example of this is an action with a finite invariant measure, see Corollary 17.

Remark 28. Theorem 23 and Corollary 24 can be extended to the orbit equivalence relation of any (not necessarily free) action, and, more generally, to an arbitrary countable non-singular equivalence relation $R$ on a Lebesgue space $(X, m)$. The arguments are precisely the same; however, in order to spare the reader the trouble of going through the definitions from the ergodic theory of equivalence relations (see [FM77]) we confined ourselves just to the case of free actions. This generality is sufficient, on one hand, to expose our (very simple) line of argument, and, on the other hand, to deal with our main application to boundary actions (Theorem 40). Here is a brief outline of the necessary modifications:

(i) The definitions of the continual and the discontinual parts of an equivalence relation $R$ remain the same as for actions (Definition 2) and are formulated in terms of the conditional measures on the ergodic components with respect to $R$;

(ii) Instead of the orbits $G x$ one has to talk about the equivalence classes $R(x)$. In particular, the definitions of conservativity and infinite conservativity of 
$R$ (based on the corresponding definitions of recurrence and infinite recurrence from Definition 9) have to be formulated in terms of the equivalence classes (for the orbit equivalence relation of an action this is the same as the strict conservativity discussed in Remark 20). Then the same argument as in Proposition 10 implies that $\mathscr{C}=$ Cons $=$ Cons $_{\infty}$.

(iii) The measures $\mu_{x}$ on the equivalence classes have to be defined in terms of the Radon-Nikodym cocycle of the equivalence relation rather than in terms of Radon-Nikodym derivatives;

(iv) The summation in formulas (25) and (26) has to be done over the equivalence class of $x$.

There is also another way of extending Theorem 23 and Corollary 24 to general (not necessarily free) actions which provides the following characterization of the infinitely conservative part:

Theorem 29. Let $G \circlearrowleft(X, m)$ be a measure class preserving action of a countable group $G$ on a Lebesgue space. Then

$$
\begin{aligned}
\text { Cons }_{\infty} & =\{x \in X: \exists t>0 \text { with } \operatorname{card}\{g \in G: \operatorname{dgm} / d m(x)>t\}=\infty\} \\
& =\left\{x \in X: \sum_{g \in G} \frac{d g m(x)}{d m(x)}=\infty\right\} .
\end{aligned}
$$

Proof. The set $X$ splits into two $G$-invariant measurable subsets $X_{<\infty}=\bigcup_{k<\infty} X_{\mathbf{k}}$ and $X_{\infty}$ determined by the size of the point stabilizers.

Let us first look at the set $X_{<\infty}$. On each of the sets $X_{\mathbf{k}}$ with $1<k<\infty$ the description (30) of Cons $\infty_{\infty} \cap X_{\mathbf{k}}$ (which coincides with $\mathscr{C} \cap X_{\mathbf{k}}$ by Theorem 14) can be proved in the same way as it was done in Theorem 23 for $X_{1}$. Further, on each of these sets $X_{\mathbf{k}}$ the sum from (31) is equal to $k$ times the sum of the values of the Radon-Nikodym cocycle along the orbit of $x$, so that these sums are finite or infinite simultaneously. Thus, in the same way as in Theorem 23 , on each set $X_{\mathbf{k}}$ condition (31) provides a description of Cons $_{\infty} \cap X_{\mathbf{k}}=\mathscr{C} \cap X_{\mathbf{k}}$.

As for the set $X_{\infty}$, its definition directly implies that $X_{\infty} \subset$ Cons $_{\infty}$ and that both conditions (30) and (31) are satisfied on the whole of $X_{\infty}$.

\section{Application to boundary actions}

2.A. Hyperbolic spaces and limit sets. Recall that a non-compact complete proper metric space $\mathscr{X}$ is Gromov $\delta$-hyperbolic (with $\delta \geq 0$ ) if its metric $d$ satisfies the $\delta$-ultrametric inequality

$$
(x \mid z)_{o} \geq \min \left\{(x \mid y)_{o},(y \mid z)_{o}\right\}-\delta \quad \forall x, y, z, o \in \mathscr{X},
$$

where

$$
(x \mid y)_{o}=\frac{1}{2}[d(o, x)+d(o, y)-d(x, y)]
$$

is the Gromov product. In addition we require that the space $X$ be separable. On the other hand, we do not require the space $\mathscr{X}$ to be geodesic. This class of spaces contains Cartan-Hadamard manifolds with pinched sectional curvatures (in particular, the classical hyperbolic spaces of constant negative curvature) and metric trees, see [Gro87, GdlH90] for more details. 
A Gromov hyperbolic space $\mathscr{X}$ admits a natural hyperbolic compactification $\overline{\mathscr{X}}=$ $\mathscr{X} \cup \partial \mathscr{X}$, and the action of the isometry group $\operatorname{Iso}(\mathscr{X})$ extends by continuity to a continuous boundary action on $\partial \mathscr{X}$. The boundary $\partial \mathscr{X}$ is a Polish space.

The limit set $\Lambda=\Lambda_{G} \subset \partial \mathscr{X}$ of a discrete subgroup $G \subset \operatorname{Iso}(\mathscr{X})$ (any such subgroup is at most countable) is the set of all the limit points of any given orbit $G o, o \in \mathscr{X}$, with respect to the hyperbolic compactification, so that the closure of the orbit $G o$ in the hyperbolic compactification is $G o \cup \Lambda_{G}$ (this definition does not depend on the choice of the basepoint $o$ ). The limit set is closed and $G$-invariant. Moreover, if the group $G$ is non-elementary (its limit set is infinite $\equiv$ contains at least 3 points), then its action on $\Lambda_{G}$ is minimal (there are no proper $G$-invariant closed subsets), whereas the action of $G$ on the complement $\partial \mathscr{X} \backslash \Lambda_{G}$ is properly discontinuous (no orbit has accumulation points) [Gro87, Bou95].

The latter result provides a topological decomposition of the boundary action. On the other hand, the situation is more complicated from the measure-theoretical point of view. Let $m$ be a purely non-atomic $G$-quasi-invariant measure on $\partial \mathscr{X}$. The complement $\partial \mathscr{X} \backslash \Lambda_{G}$ is obviously contained in the discontinual part of the action. However, this is as much as can a priori be said about the ergodic properties of the boundary action. In particular, the action on $\Lambda_{G}$ need not be ergodic or conservative. There are numerous examples witnessing to this; see [GKN09] for a detailed discussion of the simplest model case of the action of a subgroup of a free group on the boundary of the ambient group and for further references.

One can specialize the type of convergence in the definition of the limit set. For instance, the radial limit set $\Lambda^{\text {rad }}$ is the set of all the accumulation points of any fixed orbit $G o, o \in \mathscr{X}$, which stay inside a tubular neighbourhood of a certain geodesic ray in $\mathscr{X}$. Yet another type of the boundary convergence, which we are going to describe below, is provided by horospheric neighborhoods.

Denote by

$$
\beta_{z}(x, y)=d(y, z)-d(x, z), \quad x, y \in \mathscr{X}
$$

the distance cocycle associated with a point $z \in \mathscr{X}$, and, following [Kai04] (cf. [Gro87, GdlH90]), put

$$
\beta_{\omega}(x, y)=\limsup _{z \rightarrow \omega} \beta_{z}(x, y) \quad \forall x, y \in \mathscr{X}, \omega \in \partial \mathscr{X}
$$

If the space $\mathscr{X}$ is CAT $(-1)$ (e.g., a Cartan-Hadamard manifold with pinched sectional curvatures or a tree), then limsup in the above formula can be replaced just with the ordinary limit, and $\beta_{\omega}$ are the boundary Busemann cocycles. Although for a general Gromov hyperbolic space $\beta_{\omega}$ are not, generally speaking, cocycles, they still satisfy the cocycle identity with a uniformly bounded error (i.e., they are quasicocycles). Namely,

Proposition 33. [Kai04] There exists a constant $C \geq 0$ depending on the hyperbolicity constant $\delta$ of the space $\mathscr{X}$ only such that for any $\omega \in \partial \mathscr{X}$ the function $\beta_{\omega}$ (32) has the following properties:

(i) $\beta_{\omega}$ is "jointly Lipschitz", i.e., $\left|\beta_{\omega}(x, y)\right| \leq d(x, y)$ for all $x, y \in \mathscr{X}$, in particular, $\beta_{\omega}(x, x) \equiv 0$

(ii) $0 \leq \beta_{\omega}(x, y)+\beta_{\omega}(y, z)+\beta_{\omega}(z, x) \leq C$ for all $x, y, z \in \mathscr{X}$. 
The quasi-cocycles $\beta_{\omega}$ are obviously invariant with respect to the isometries of $\mathscr{X}$, i.e.,

$$
\beta_{g \omega}(g x, g y)=\beta_{\omega}(x, y) \quad \forall x, y \in \mathscr{X}, \omega \in \partial \mathscr{X}, g \in \operatorname{Iso}(\mathscr{X}) .
$$

We shall define the horoball in $\mathscr{X}$ centered at a boundary point $\omega \in \partial \mathscr{X}$ and passing through a point $o \in \mathscr{X}$ as

$$
\operatorname{HBall}_{\omega}(o)=\left\{x \in \mathscr{X}: \beta_{\omega}(o, x) \leq 0\right\} .
$$

Definition 34. The big (resp., small) horospheric limit set $\Lambda^{\text {horB }}=\Lambda_{G}^{\text {horB }}$ (resp., $\Lambda^{\text {horS }}=\Lambda_{G}^{\text {horS }}$ ) of a discrete group $G$ of isometries of a Gromov hyperbolic space $\mathscr{X}$ is the set of all the points $\omega \in \partial \mathscr{X}$ such that a certain (resp., any) horoball centered at $\omega$ contains infinitely many points from a fixed orbit $G o, o \in \mathscr{X}$ (the resulting set does not depend on the choice of the orbit Go, see Remark 35 below).

Remark 35. As it follows from Proposition 33, for any fixed reference point $o \in \mathscr{X}$ the big (resp., small) horospheric limit sets can also be defined as the set of all the points $\omega \in \partial \mathscr{X}$ for which the set

$$
\left\{x \in G o: \beta_{\omega}(o, x) \leq t\right\}
$$

is infinite for a certain (resp., for any) $t \in \mathbf{R}$.

Remark 36. Usually our small horospheric limit set is called just the horospheric limit set, and in the context of Fuchsian groups its definition, along with the definition of the radial limit set, goes back to Hedlund [Hed36]. Following [Mat02] (in the Kleinian case) we call it small in order to better distinguish it from the big one, which, although apparently first explicitly introduced by Tukia [Tuk97] (again just in the Kleinian case), essentially appears (for Fuchsian groups) already in Pommerenke's paper [Pom76].

The horospheric limit sets $\Lambda^{\text {horS }}, \Lambda^{\text {horB }}$ are obviously $G$-invariant, Borel, and contained in the full limit set $\Lambda$ (because the only boundary accumulation point of any horoball is just its center).

\section{B. Boundary conformal streams.}

Definition 37. A family of pairwise equivalent finite measures $\lambda=\left\{\lambda_{x}\right\}$ on the boundary $\partial \mathscr{X}$ of a Gromov hyperbolic space $\mathscr{X}$ parameterized by points $x \in \mathscr{X}$ is called a quasi-conformal stream of dimension $D>0$ if there exists a constant $C>0$ such that

$$
\left|\log \frac{d \lambda_{x}}{d \lambda_{y}}(\omega)-D \beta_{\omega}(x, y)\right| \leq C \quad \forall x, y \in \mathscr{X}, \omega \in \partial \mathscr{X} .
$$

A stream $\lambda$ is invariant with respect to a group $G \subset \operatorname{Iso}(\mathscr{X})$ if

$$
\lambda_{g x}=g \lambda_{x} \quad \forall g \in G, x \in \mathscr{X} .
$$

Remark 38. We follow here the terminology developed in [KL05]. More traditionally, any invariant quasi-conformal stream is determined just by a single finite boundary quasi-conformal measure $\lambda=\lambda_{o}$ with the property that

$$
\left|\frac{d g \lambda}{d \lambda}(\omega)-D \beta_{\omega}(g o, o)\right| \leq C \quad \forall g \in G, \omega \in \partial \mathscr{X} .
$$

for a certain reference point $o \in \mathscr{X}$. If $\beta_{\omega}$ are cocycles (which is the case for $\operatorname{CAT}(-1)$ spaces), then any measure $\lambda$ satisfying (39) is equivalent to a unique finite measure $\lambda^{\prime}$ (called conformal) which satisfies formula (39) with $C=0$ (it follows from the 
fact that any uniformly bounded cocycle is cohomologically trivial). This definition is motivated by the fact that the visual measure on the boundary sphere $\partial \mathbf{H}^{d+1}$ of the classical $(d+1)$-dimensional hyperbolic space with sectional curvature -1 is conformal of dimension $d$ (in our terminology it means that the visual stream which consists in assigning to any point from the hyperbolic space the associated visual measure is conformal). However, the limit set of the group can be "much smaller" than the boundary sphere and be a null set with respect to the visual measure. Existence of conformal measures which are concentrated on the limit set (and for which the dimension coincides with the critical exponent of the group) was first established by Patterson [Pat76] in the case of Fuchsian groups. His construction was further generalized (see [Sul79], [Kai90]), ultimately providing existence of a conformal measure for any closed subgroup of isometries of a general CAT(-1) space [BM96]. For discrete isometry groups of general Gromov hyperbolic spaces existence of measures satisfying (39) (i.e., existence of invariant quasi-conformal streams in our terminology) was established by Coornaert [Coo93] (also by a generalization of Patterson's construction).

2.C. Hopf decomposition of the boundary action. We are now ready to proceed to the main application of Theorem 23 which is a description of the Hopf decomposition of the boundary action of a discrete subgroup $G \subset \operatorname{Iso}(\mathscr{X})$ with respect to (the measure class of) a quasi-conformal invariant stream.

As the atomic part of such a stream is obviously discontinual (so that by Theorem 14 its Hopf decomposition is completely determined by the size of the point stabilizers), we can restrict our considerations to the purely non-atomic case only. We shall use the notation introduced in Section 1.C for the subsets of $\partial \mathscr{X}$ determined by the size of their $G$-stabilizers.

Theorem 40. Let $G$ be a discrete group of isometries of a Gromov hyperbolic space $\mathscr{X}$, and $\lambda$ be a purely non-atomic $G$-invariant quasi-conformal boundary stream. Then

(i) The infinitely conservative part of the action is $(\bmod 0)$ Cons $_{\infty}=\Lambda_{G}^{\text {horB }}$;

(ii) The conservative part of the action of $G$ on the hyperbolic boundary $\partial \mathscr{X}$ with respect to the measure class of $\lambda$ is $(\bmod 0)$ Cons $=\Lambda_{G}^{\text {horB }} \cup \partial \mathscr{X}_{>\mathbf{1}}$, and its dissipative part is $(\bmod 0)$ Diss $=\partial \mathscr{X} \backslash$ Cons $=\partial \mathscr{X}_{\mathbf{1}} \backslash \Lambda_{G}^{\text {horB }}$.

Proof. Fix a reference point $o \in \mathscr{X}$ and consider the associated measure $\lambda_{o}$. Without loss of generality we may assume that it is normalized, so that $\left(\partial \mathscr{X}, \lambda_{o}\right)$ is a Lebesgue space. Since the action of $G$ on $\mathscr{X}$ is properly discontinuous, the $G$ stabilizer of the point $o$ is at most finite. Then, in view of (39) and Remark 35, the description of Cons $_{\infty}$ immediately follows from Theorem 29, whereas the description of Cons follows from Corollary 15.

Theorem 40(i) in combination with Theorem 29 implies

Corollary 41. The big horospheric limit set $\Lambda_{G}^{\text {horB }}$ coincides $(\bmod 0)$ with the divergence set of the Poincaré-Busemann series

$$
\left\{\omega \in \partial \mathscr{X}: \sum_{g \in G} e^{D \beta_{\omega}(g o, o)}=\infty\right\}
$$

Remark 42. Since the stream $\lambda$ is purely non-atomic, the set of fixed points of hyperbolic and parabolic elements of $G$ is at most countable, and therefore it is 
$\lambda$-negligible. On the other hand, the set of fixed points of an elliptic element may be quite big and may even coincide with the whole boundary $\partial \mathscr{X}$. For the simplest example take a finite group $G_{0}$, and let $\mathscr{X}^{\prime}=G_{0} \times \mathscr{X}$. The space $\mathscr{X}^{\prime}$ is hyperbolic simultaneously with the space $X$, and $G^{\prime}=G_{0} \times G$ is a discrete $\operatorname{subgroup}$ of $\operatorname{Iso}\left(\mathscr{X}^{\prime}\right)$ provided $G$ is a discrete subgroup of $\operatorname{Iso}(\mathscr{X})$. Then the action of the group $G_{0} \subset G^{\prime}$ on the hyperbolic boundary $\partial \mathscr{X}^{\prime} \cong \partial \mathscr{X}$ is trivial.

Remark 43. The infinitely conservative part Cons ${ }_{\infty}$ coincides with the continual part $\mathscr{C}$ of the boundary action on the set $\partial \mathscr{X}_{<\infty}$. On the other hand, they need not coincide on the set $\partial \mathscr{X}_{\infty}$. An easy modification of the above arguments shows that $\mathscr{C} \cap \partial \mathscr{X}_{\infty}$ is $(\bmod 0)$ the set of points $\omega \in \mathscr{X}_{\infty}$ for which a certain horoball centered at $\omega$ contains infinitely many $G_{\omega}$-orbits. By discarding fixed points of hyperbolic and parabolic elements we may assume that $G_{\omega}$ is an infinite torsion group.

It is easy to construct an example of an infinite torsion group embedded as a discrete parabolic subgroup in the group of isometries of a Gromov hyperbolic space (I am grateful to I. Kapovich for a discussion of this topic). Let $\mathscr{X}$ be the homogeneous tree of degree $d+1$ endowed with the graph metric. Fix a vertex $o \in \mathscr{X}$, a boundary point $\omega \in \partial \mathscr{X}$, and let $\mathscr{L}: \mathscr{X} \rightarrow \mathbf{Z}_{d}$ be a labelling of vertices of $\mathscr{X}$ with elements of the cyclic group $\mathbf{Z}_{d}$ such that for any vertex $x \in \mathscr{X}$ all the $d$ neighbours $y$ of $x$ with $\beta_{\omega}(x, y)=1$ (the ones which are "further" from $\omega$ ) are labelled differently. Then any point $x \in \mathscr{X}$, by reading the labels along the geodesic ray $\gamma=[x, \omega)$ according to the formula $\varepsilon_{k}=\mathscr{L}\left(\gamma\left(\beta_{\omega}(o, x)-k\right)\right)$, gives rise to the associated sequence of labels $\left(\varepsilon_{k}\right)_{k \leq \beta_{\omega}(o, x)}$. By using this correspondence one can now define, for any integer $k$, the group $G_{k} \cong \mathbf{Z}_{d}$ of isometries of $\mathscr{X}$ : it cyclically changes the symbol $\varepsilon_{k}$ in the above sequence (provided $\beta(o, x) \geq k$; otherwise the action of $G_{k}$ is trivial). Obviously, the groups $G_{k}$ commute, so that the direct sum $G=\sum_{k \leq 0} G_{k}$ is an infinite torsion group. The group $G$ fixes the boundary point $\omega$; on the other hand, it is not hard to see that $G$ is discrete.

However, it is highly unlikely that the set $\partial \mathscr{X}_{\infty}$, or, equivalently, the set of points $\omega \in \partial \mathscr{X}$ whose stabilizer $G_{\omega}$ is an infinite torsion group, may be uncountable, let alone be non-negligible with respect to a quasi-conformal invariant stream.

Remark 44. For Fuchsian groups with respect to the visual stream on the boundary circle Theorem 40 and Corollary 41 were proved by Pommerenke [Pom76] (although in a somewhat different terminology, see the discussion in [Pom82, Section 1]). Pommerenke's argument uses analytic properties of the Blaschke products and does not immediately carry over to the higher dimensional situation. Sullivan [Sul81] used a more direct geometrical approach and established Theorem 40 for Kleinian groups, again with respect to the visual stream (actually he considered the small horospheric limit set $\Lambda^{\text {horS }}$ essentially showing that $\Lambda^{\text {horB }} \backslash \Lambda^{\text {horS }}$ is a null set, see Remark 45 below). By extending Sullivan's approach (with some technical complications) Tukia [Tuk97] proved Theorem 40 for Kleinian groups with respect to an arbitrary invariant conformal stream. In all these particular cases the set $\partial \mathscr{X}_{>1}$ is negligible, so that Cons $=$ Cons $_{\infty}=\mathscr{C}$.

Remark 45. Our argument used the characterization of the infinitely conservative part of an action $G \circlearrowleft(X, m)$ as the set of all the points $x \in X$ such that for a certain $t>0$

$$
\{g \in G: d g m / d m(x)>t\} \quad \text { is infinite }
$$


(Theorem 29), which in our setup is precisely the big horospheric limit set $\Lambda^{\text {horB }}$. The small horospheric limit set $\Lambda^{\text {horS }}$ corresponds to requiring that the above condition hold for any $t>0$, which, in general, is not equivalent to conservativity (see Remark 27). From this point of view the right object in the context of studying conservativity of boundary actions is definitely $\Lambda^{\text {horB }}$ rather than $\Lambda^{\text {horS }}$. The difference $\Lambda^{\text {horB }} \backslash \Lambda^{\text {horS }}$ is the set of all the boundary points $\omega \in \partial \mathscr{X}$ for which among the horoballs centered at $\omega$ there are both ones containing finitely many points from the orbit $G o$ of a fixed reference point $o \in \mathscr{X}$ and ones containing infinitely many points from Go. Sullivan [Sul81] essentially proved that $\Lambda^{\text {horB }} \backslash \Lambda^{\text {horS }}$ is a null set with respect to the visual stream for Kleinian groups (also see the discussion of his result in [Pom82, Section 1]); for subgroups of a free group (again with respect to the uniform stream on the boundary of the ambient group) it was done in [GKN09]. We are not aware of any other results of this kind; in particular, it is already not known for Kleinian groups with respect to general invariant conformal streams, see [Tuk97]. Nonetheless, it seems plausible that $\Lambda^{\text {horB }} \backslash \Lambda^{\text {horS }}$ is a null set with respect to any invariant quasi-conformal stream on an arbitrary Gromov hyperbolic space.

\section{References}

[Aar97] Aaronson, J.: An introduction to infinite ergodic theory. - Math. Surveys Monogr. 50, Amer. Math. Soc., Providence, RI, 1997.

[BM96] Burger, M., and S. Mozes: CAT(-1)-spaces, divergence groups and their commensurators. - J. Amer. Math. Soc. 9:1, 1996, 57-93.

[Bou95] Bourdon, M.: Structure conforme au bord et flot géodésique d'un CAT(-1)-espace. Enseign. Math. (2) 41:1-2, 1995, 63-102.

[CFS82] Cornfeld, I. P., S. V. Fomin, and Ya. G. Sină̌: Ergodic theory. - Grundlehren Math. Wiss. 245, transl. from the Russian by A. B. Sosinskiǔ, Springer-Verlag, New York, 1982.

[Coo93] Coornaert, M.: Mesures de Patterson-Sullivan sur le bord d'un espace hyperbolique au sens de Gromov. - Pacific J. Math. 159:2, 1993, 241-270.

[FM77] Feldman, J., and C. C. Moore. Ergodic equivalence relations, cohomology, and von Neumann algebras. I. - Trans. Amer. Math. Soc. 234:2, 1977, 289-324.

[GdlH90] Ghys, É., and P. DE LA HARPe (eds.): Sur les groupes hyperboliques d'après Mikhael Gromov. - Progr. Math. 83, papers from the Swiss Seminar on Hyperbolic Groups held in Bern, 1988, Birkhäuser Boston Inc., Boston, MA, 1990.

[GKN09] Grigorchuk, R., V. Kaimanovich, and T. Nagnibeda: Ergodic properties of boundary actions and Nielsen-Schreier theory. - arXiv:0901.4734, 2009.

[Gro87] Gromov, M.: Hyperbolic groups. - In: Essays in group theory, Math. Sci. Res. Inst. Publ. 8, Springer, New York, 1987, 75-263.

[Hed36] Hedlund, G. A.: Fuchsian groups and transitive horocycles. - Duke Math. J. 2:3, 1936, $530-542$.

[Hop30] Hopf, E.: Zwei Sätze über den wahrscheinlichen Verlauf der Bewegungen dynamischer Systeme. - Math. Ann. 103:1, 1930, 710-719.

[Kai90] Kaimanovich, V. A.: Invariant measures of the geodesic flow and measures at infinity on negatively curved manifolds. - Ann. Inst. H. Poincaré Phys. Théor. 53:4, 1990, 361-393.

[Kai04] Kaimanovich, V. A.: Boundary amenability of hyperbolic spaces. - In: Discrete geometric analysis, Contemp. Math. 347, Amer. Math. Soc., Providence, RI, 2004, 83-111.

[KL05] Kaimanovich, V. A., and M. Lyubich: Conformal and harmonic measures on laminations associated with rational maps. - Mem. Amer. Math. Soc. 173:820, 2005. 
[Kre85] Krengel, U.: Ergodic theorems. - de Gruyter Stud. Math. 6, with a supplement by Antoine Brunel, Walter de Gruyter \& Co., Berlin, 1985.

[Mat02] Matsuzaki, K.: Conservative action of Kleinian groups with respect to the PattersonSullivan measure. - Comput. Methods Funct. Theory 2:2, 2002, 469-479.

[Pat76] Patterson, S. J.: The limit set of a Fuchsian group. - Acta Math. 136:3-4, 1976, 241273.

[Pom76] Pommerenke, Ch.: On the Green's function of Fuchsian groups. - Ann. Acad. Sci. Fenn. Ser. A I Math. 2, 1976, 409-427.

[Pom82] Pommerenke, Ch.: On Fuchsian groups of accessible type. - Ann. Acad. Sci. Fenn. Ser. A I Math. 7:2, 1982, 249-258.

[Roh52] Rohlin, V. A.: On the fundamental ideas of measure theory. - Amer. Math. Soc. Transl. 1952:71, 1952, 1-55.

[Sch77] Schmidt, K.: Cocycles on ergodic transformation groups. - Macmillan Lectures in Math. 1, Macmillan Company of India, Ltd., Delhi, 1977.

[Sul79] Sullivan, D.: The density at infinity of a discrete group of hyperbolic motions. - Inst. Hautes Études Sci. Publ. Math. 50, 1979, 171-202.

[Sul81] Sullivan, D.: On the ergodic theory at infinity of an arbitrary discrete group of hyperbolic motions. - In: Riemann surfaces and related topics: Proceedings of the 1978 Stony Brook Conference (State Univ. New York, Stony Brook, N.Y., 1978) (Princeton, N.J.), Ann. of Math. Stud. 97, Princeton Univ. Press, 1981, 465-496.

[Tuk97] TukiA, P.: Conservative action and the horospheric limit set. - Ann. Acad. Sci. Fenn. Math. 22:2, 1997, 387-394.

Received 11 July 2008 